\title{
Mining Data Streams using Option Trees
}

\author{
B.Reshma Yusuf, Dr.P.Chenna Reddy \\ JNTUA college of engineering, pulivendula, Andhra Pradesh, INDIA. \\ resh.yusuf@gmail.com,pcreddy1@rediffmail.com
}

\begin{abstract}
In today's applications, evolving data streams are stored as very large databases; the databases which grow without limit at a rate of several million records per day. Data streams are ubiquitous and have become an important research topic in the last two decades. Mining these continuous data streams brings unique opportunities, but also new challenges. For their predictive nonparametric analysis, Hoeffding-based trees are often a method of choice, which offers a possibility of any-time predictions. Although one of their main problems is the delay in learning progress due to the presence of equally discriminative attributes. Options are a natural way to deal with this problem. In this paper, Option trees which build upon regular trees is presented by adding splitting options in the internal nodes to improve accuracy, stability and reduce ambiguity. Results based on accuracy and processing speed of algorithm under various memory limits is presented. The accuracy of Hoeffding Option tree with Hoeffding trees under circumstantial conditions is compared.
\end{abstract}

Index Terms - Data streams, hoeffding trees, option trees, large databases

\section{INTRODUCTION}

Data mining is the task of discovering interesting and hidden patterns from large amounts of data where the data can be stored in databases, data warehouses, OLAP (on line analytical process ) or other repository information. At present the most efficient algorithms available are focused on making it possible to mine databases that do not fit in main memory by only requiring sequential scans of the disk. But these algorithms are tested only up to a few million examples. In many applications this is less than a day's worth of data. For example, every day, retail chains record millions of transactions, telecommunications companies connect millions of calls, large banks process millions of ATM and credit card operations, and popular Web sites $\log$ millions of hits. As the expansion of the Internet continues and ubiquitous computing becomes a reality, we can expect that such data volumes will become the rule rather than the exception. Current data mining systems are not suitable for such type of data as the amount of data generated and accumulated is rapidly increasing. To this extent, a new mining application that accepts data streams as input has emerged which can be expressed as stream mining.
A data stream [1] is an ordered sequence of instances with bounded main memory and data may be evolving over time at a higher rate than they can be mined. Even simply storing the examples for future use may be lost or corrupted and can become unusable when the relevant information is no longer available.

The contribution of this paper is to design a decision tree learner for extremely large (potentially infinite) datasets. Such a decision tree learner should require each example to be read at most once, and only a small constant time to process it. This will make it possible to directly mine online data sources (i.e., without ever storing the examples), and to build potentially very complex trees with acceptable computational cost. The problem of deciding exactly how many examples are necessary at each node is solved by using a statistical result known as the Hoeffding bound [2] (or additive Chernoff bound).

Consider a real-valued random variable $r$ whose range is $\mathrm{R}$ (e.g., for a probability the range is one, and for an information gain the range is $\log \mathrm{c}$, where $\mathrm{c}$ is the number of classes). Suppose we have made n independent observations of this variable, and computed their mean $\mathrm{r}$.

Hoeffding bound states that, with probability $1-\delta$, the true mean of the variable is at least $r^{\prime}-\varepsilon$, where

$$
\varepsilon=\sqrt{\frac{R^{2} \ln (1 / \delta)}{2 n}}
$$

Hoeffding-based tree learners have been recognized as the most efficient in terms of processing speed per example, although their learning might be slow, which results in lower any-time accuracy at the beginning. The Hoeffding trees tend to be less accurate in situations where several attributes appear to be equally discriminative. In such tie-situations, the split selection method based on the Hoeffding bound might never decide, which attribute is significantly better. To solve this problem, Hoeffding-based tree learners typically use a tie-breaking mechanism based on a user-defined threshold, which implicitly specifies the amount of data that has to be observed in order to decide on one of the competitive attributes. Setting this threshold requires knowledge of the problem domain. An additional side effect is that the splitting decision will be significantly delayed, which results in a lower any-time accuracy during this delay.

This problem is solved using option trees, which can include option nodes in addition to ordinary split nodes. 
The main motivation is that introducing option nodes removes the need for selecting the best splitting attribute. Main idea is to introduce options only when splitting decisions are ambiguous, which will avoid excessive and unnecessary tree growth and reduce memory consumption. We opt for a faster improvement of the any-time accuracy, which is a very important property of predictive algorithms for real-time dynamic systems.

\section{RELATED WORK}

Classification is one of the most familiar and most popular data mining techniques. It aims to find a function that can map each data item in dataset into one of several predefined classes. Many classification algorithms are available in literature but decision trees is most commonly used because of its ease of implementation and easier to understand compared to other classification algorithms.

Decision tree is one of the most often used techniques in the data mining literature. Each node of a decision tree contains a test on an attribute. Each branch from a node corresponds to a possible outcome of the test and each leaf contains a class prediction. A decision tree is constructed by recursively replacing leaves by test nodes, starting at the root. The attribute to test in a leaf is chosen by comparing all available attributes and choosing the best one according to some heuristic evaluation function.

Classic decision tree learners like ID3, C4.5, and CART assume that all training examples can be stored simultaneously in memory, and thus are severely limited in the number of examples from which they can learn.

Previous work on scaling up decision tree learning produced systems such as SLIQ [3], SPRINT [4]. These systems perform batch learning of decision trees from large data sources in limited memory by performing multiple passes over the data and using external storage. Such operations are not suitable for high speed stream processing.

Hoeffding tree an incremental decision tree algorithm proposed by Domingos and Hulten in the paper "Mining High Speed Data Streams". For streams made up of discrete types of data, Hoeffding bounds guarantee that the output model is asymptotically nearly identical to that of a conventional decision tree. The Hoeffding tree algorithm is the basic theoretical algorithm, while VFDT adopts several enhancement techniques for practical applications, such as grace period, pre-pruning, and tie breaking.

Hulten proposed a new algorithm called CVFDT [5], which used a fixed-size window to determine which nodes are aging and may need updating. For fragments of the Hoeffding tree that become old and inaccurate, alternative subtrees are grown that later replace the outdated nodes. It is worth noting, that the whole process does not require model retraining. Outdated examples are forgot by updating node statistics and necessary model changes are performed on subtrees rather than the whole classifier.

Some methods for voting classification algorithms have been developed to be very successful in improving accuracy of certain classifiers for artificial and real world datasets. They are based on the performance of previous classifier.

Option decision trees [6] are used to reduce the error of decision trees on real world problems by combining multiple options which is quite similar to that of voting algorithms that learn multiple models and combine the predictions. The main goal of the paper is to explore when option nodes are most useful and to control the growth of trees by which complexity of little utility is limited.

Option nodes are also used in the context of learning from data streams, as an extension of Hoeffding trees [7] for classification. Although Hoeffding trees are more stable than batch tree learners, decisions are still subject to limited lookahead. This is the main motivation of Pfahringer et al. Their approach is somewhat different from the proposed batch ones, mainly because option nodes are not introduced in the split selection process, but only after a node has been transformed into an internal (decision) node

Kirkby [8] proposed an Option Tree that allows each training example to update a set of option nodes rather than just a single leaf. Option nodes work like standard decision tree nodes with the difference that they can split the decision paths into several sub trees. Making a decision with an option tree involves combining the predictions of all applicable leaves into a single result.

Hoeffding tree algorithm is clearly explained and experiments are conducted concerning the windowed classifier, hoeffding option tree and hoeffding tree with a drift detector in mining data streams using concept drift [9]. The results of hoeffding option tree are always more accurate than the single hoeffding tree with a drift detector.

\section{OPTION TREES}

Option trees are a single general structure making it possible to travel down multiple paths and arrive at multiple leaves. This is achieved by introducing the possibility of option nodes to the tree, alongside the standard decision nodes and leaf nodes.

An option node [4] splits the decision path several ways - when an option node is encountered several different sub trees are traversed, which could themselves contain more option nodes, thus the potential for reaching different leaves is multiplied by every option. Making a decision with an option tree involves combining the predictions of the applicable leaves into a final result. A potential benefit of option trees over a traditional ensemble is that the more flexible representation can save space.

Consider as an extreme example an ensemble of one hundred mostly identical large trees, where the only 
difference between each tree lies at a single leaf node, in the same position in each tree. The standard ensemble representation would require one hundred whole copies of the tree where only the leaf would differ. Efficiently represented as an option tree this would require almost a hundred times less space, where the varying leaf could be replaced by an option node splitting one hundred ways leading to the one hundred different leaf variants.

\section{Methodology}

Massive Online Analysis (MOA) is a software environment for implementing algorithms and running experiments for online learning. It is designed to deal with the problems of scaling up the implementation of state of the art algorithms to real world dataset sizes and of making algorithms comparable in benchmark streaming settings. It is implemented in Java and contains a collection of data stream generators, online learning algorithms, and evaluation procedures.

\section{A. Environments:}

Three environments are simulated using memory limits, since memory limits cannot be ignored and can significantly limit capacity to learn from data streams. Potential practical deployment of data stream classification has been divided into scenarios of increasing memory utilization, from the restrictive sensor environment, to a typical consumer grade handheld PDA environment, to the least restrictive environment of a dedicated server.

\section{1) Sensor Network}

This environment represents the most restrictive case, learning in 100 kilobytes of memory. Because this limit is so restrictive, it is an interesting test case for algorithm efficiency.

When memory limits are in the order of kilobytes, other applications requiring low memory usage also exist, such as specialized hardware in which memory is expensive.

\section{2) Handheld Computer}

In this case the algorithm is allowed 32 megabytes of memory. This simulates the capacity of lightweight consumer devices designed to be carried around by users and can easily fit into a shirt pocket. The ability to do analysis on site with a handheld device is desirable for certain applications.

\section{3) Server}

This environment simulates either a modern laptop/desktop computer or server dedicated for processing a data stream. The memory limit assigned in this environment is 400 megabytes. Considering that several algorithms have difficulty in fully utilizing this much working space, it seems sufficiently realistic to impose this limit.

There are many applications that fit into this higher end of the computing scale. An obvious task is analyzing data arising from the Internet, as web searches, web usage, site logs or click streams. Smaller scale computer networks also produce traffic of interest, as do other telecommunication activities, phone call logs for example. Banks may be interested in patterns of ATM transactions, and retail chains and online stores will need details about customer purchases.

\section{B. Data generators:}

MOA stream generators allow simulating potentially infinite sequence of data. The following are the generators used for generating data according to some pattern instead of reading data from file, database or any other data source.

\section{1) Random Tree Generator}

Random tree generator generates examples by assigning uniformly distributed random values to attributes which then determine the class label via the tree which choose attributes at random to split. The generator has parameters to control the number of classes, attributes and depth of tree. Two random trees were generated one is simple and the other complex.

The simple random tree (rts) has ten nominal attributes with five values each, ten numeric attributes, two classes, a tree depth of five, with leaves starting at level three and a 0.15 chance of leaves. The complex random tree (rtc) has 50 nominal attributes with five values each, 50 numeric attributes, two classes, a tree depth of ten, with leaves starting at level five and a 0.15 chance of leaves.

A degree of noise can be introduced to the examples after generation. The streams rtsn and rtcn are introduced by adding $10 \%$ noise to the respective random tree data streams.

\section{2) Random RBF Generator}

Random RBF generates examples from a fixed number of random centroids. Each time a centroid is selected at random, a data point around the centroid is drawn randomly. The centroid determines the class label of the example, and the data point determines the attributes of the example. Examples in Random Tree are generated by assigning random values to each attribute first and the class label is determined via a preconstructed decision tree. Only numeric attributes are generated. Two random streams are produced one simple and other complex.

The simple RBF (RRBFS) generator has 100 centers and ten attributes where complex RBF (RRBFC) generator has 50 attributes and 1000 centers. Both have only two classes.

\section{3) LED Generator}

The generator actually generates stream predicting the digit displayed on a seven-segment LED display, where each attribute has a $10 \%$ chance of being inverted. It has an optimal Bayes classification rate of $74 \%$. The particular configuration of the generator used for experiments produces 24 binary attributes, 17 of which are irrelevant.

\section{4) Waveform Generator}

This generator shares its origins with LED. The goal of the task is to differentiate between three different classes of waveform, each of which is generated from a combination of two or three base waves. There are two versions of the problem. Wave21 has 21 numeric 
attributes, all of which include noise. Wave40 introduces an additional 19 irrelevant attributes.

\section{5) Function Generator}

Function generator produces a stream containing nine attributes, six numeric and three categorical which was used as data source for work on scaling up decision tree learners. There are ten functions defined for generating binary class labels from the attributes. For the experiments the ten functions are used, with a perturbation factor of $5 \%$ (referred to as genF1-genF10). Perturbation shifts numeric attributes from their true value, adding an offset drawn randomly from a uniform distribution, the range of which is a specified percentage of the total value range.

\section{EVALUATION}

Wide variety of data sets is used for evaluation. The experiments are performed on a machine $2.40 \mathrm{GHz}$ Intel Core 2 Duo processor, 2 GB RAM, running windows XP. The evaluation procedure of a learning algorithm determines which examples are used for training the algorithm, and which are used to test the model output by the algorithm. The evaluation methodology used was predictive sequential which is known as prequential approach. Prequential evaluation provides a learning curve that monitors the evaluation of learning as a process. It is based on the idea that statistical methods should be assessed by means of validity of predictions that flow from them and that such assessments can usefully be extracted from a sequence of realized data values, by forming, at each intermediate time point, a forecast for next value, based on an analysis of earlier values.

The first, and baseline algorithm is a single Hoeffding tree, enhanced with majority class prediction(HTMC) and hoeffding tree with adaptive naïve bayes leaf predictions(HTNBA) and the Hoeffding Option Tree algorithm(HOT) for which maximum of five option paths are given.

MOA framework is used for evaluating the algorithms, in which parameter settings are made.

The following are the list of parameters used for evaluating hoeffding tree algorithm:

Split confidence: The allowable error in split decision, values closer to 0 will take longer to decide. The default value is set as $10^{\wedge}-7$.

Tie threshold: A situation may occur where two or more competing attributes cannot be separated. Even with very small Hoeffding bound, it would not be able to separate them and the tree growth would stall.

Threshold below which a split will be forced to break ties. The default value is set as 0.05 .

Grace period: The number of instances a leaf should observe between split attempts. The default value is set as 200.

Pre Pruning: Pre pruning is carried out by considering at each node a NULL attribute $\mathrm{X} 0$, which consists of not splitting the node. The split will only be made if, with confidence $1-\delta$, the best split found is better according to $\mathrm{G}$ than not splitting. $\mathrm{X} 0$ will determine the leaf nodes.

HTNBA have all the parameters set as HTMC except prediction strategy. It allows either adaptive hybrid or naïve bayes prediction where HTMC allows only Majority class prediction.

In hoeffding option tree (HOT) with five option paths all the above parameters are considered along with an important parameter of HOT5 algorithm.

Secondary split confidence: The allowable error in secondary split decisions, values closer to 0 will take longer to decide. The default value is set as 0.99 .

MOA environment is used for obtaining results from the learning algorithms i.e., Hoeffding option tree algorithm and Hoeffding tree algorithm with some parameters set for evaluation using prequential evaluation technique. Data generator is chosen and algorithm is invoked for number of examples based on evaluation method and results signifying accuracy, tree size, number of leaves, etc are produced.

Comparison between Hoeffding Tree algorithm with Naïve Bayes prediction and Majority class prediction at leaves along with Hoeffding Option Tree algorithm with five option paths is done by setting the parameters given above with specified number of examples.

TABLE I

COMPARISON OF ACCURACY FOR HOEFFDING TREES WITH MAJORITY CLASS AND NAÏVE BAYES PREDICTION AT LEAVES

\begin{tabular}{|l|l|l|l|l|l|l|}
\hline \multirow{2}{*}{ Method } & \multicolumn{3}{|c|}{ htmc } & \multicolumn{3}{c|}{ htnba } \\
\hline \multirow{2}{*}{ Dataset } & $\begin{array}{l}100 \\
\text { KB }\end{array}$ & $\begin{array}{l}32 \\
\text { MB }\end{array}$ & $\begin{array}{l}400 \\
\text { MB }\end{array}$ & $\begin{array}{l}100 \\
\text { KB }\end{array}$ & $\begin{array}{l}32 \\
\text { MB }\end{array}$ & MB \\
\hline rts & 97.2 & 99.8 & 99.8 & 97.2 & 99.9 & 99.9 \\
\hline rtsn & 74.9 & 77.3 & 77.3 & 72.9 & 77.8 & 78.1 \\
\hline rtc & 77.8 & 68.1 & 68.1 & 63.5 & 69.2 & 69.2 \\
\hline rtcn & 56 & 58.9 & 58.9 & 56.0 & 59.3 & 59.3 \\
\hline rrbfs & 88 & 90 & 90 & 88 & 91.7 & 91.7 \\
\hline rrbfc & 92.1 & 95.3 & 95.3 & 92.1 & 97.3 & 97.3 \\
\hline wave21 & 81.9 & 83.1 & 83.1 & 81.5 & 86.7 & 86.7 \\
\hline wave40 & 82.1 & 83.1 & 83.1 & 82.1 & 86 & 86 \\
\hline Led & 74.9 & 75.8 & 75.8 & 75.3 & 75.2 & 75.2 \\
\hline genF1 & 95.5 & 95.5 & 95.5 & 95.5 & 95.5 & 95.5 \\
\hline genF2 & 81.1 & 85.4 & 85.4 & 81.1 & 86.5 & 86.5 \\
\hline genF3 & 97.8 & 97.8 & 97.8 & 97.8 & 97.8 & 97.8 \\
\hline genF4 & 94 & 94.4 & 94.4 & 94.1 & 94.3 & 94.3 \\
\hline genF5 & 86.8 & 91.9 & 91.9 & 86.8 & 93.1 & 93.1 \\
\hline genF6 & 88.9 & 90.8 & 90.8 & 88.9 & 91.2 & 91.2 \\
\hline genF7 & 96 & 95.9 & 95.9 & 96.2 & 97.2 & 97.2 \\
\hline genF8 & 99.4 & 99.4 & 99.4 & 99.5 & 99.4 & 99.4 \\
\hline genF9 & 95.2 & 96.5 & 96.5 & 95.2 & 96.4 & 96.4 \\
\hline genF10 & 99.7 & 99.7 & 99.7 & 99.7 & 99.7 & 99.7 \\
\hline
\end{tabular}


Result of accuracy of Hoeffding tree which uses majority class and naïve bayes at the leaves to predict the attribute are presented.

Hoeffding tree with majority class prediction gives more accurate values when compared with hoeffding tress with adaptive naïve bayes prediction at only restricted $100 \mathrm{~KB}$ environments. In the remaining two environments HTNBA is comparatively more accurate than HTMC.

The 32MB memory limit gives approximately good accuracy results in both evaluations. The datasets with noise generated give less accuracy. When a random tree is complex it gives very low accuracy results mainly in $100 \mathrm{~KB}$ environment.

TABLE II

COMPARISON OF ACCURACY FOR HOEFFDING TREES AND HOEFFDING OPTION TREES WITH FIVE OPTION PATHS

\begin{tabular}{|c|c|c|c|c|c|c|}
\hline Method & \multicolumn{3}{|c|}{$\begin{array}{l}\text { htnba } \\
\text { memory limit }\end{array}$} & \multicolumn{3}{|c|}{$\begin{array}{c}\text { hot5 } \\
\text { memory limit }\end{array}$} \\
\hline dataset & $\begin{array}{l}100 \\
\mathrm{~KB}\end{array}$ & $\begin{array}{l}32 \\
\mathrm{MB}\end{array}$ & $\begin{array}{l}400 \\
\mathrm{MB}\end{array}$ & $\begin{array}{l}100 \\
\mathrm{~KB}\end{array}$ & $\begin{array}{l}32 \\
\mathrm{MB}\end{array}$ & $\begin{array}{l}400 \\
\mathrm{MB}\end{array}$ \\
\hline rts & 97.2 & 99.9 & 99.9 & 98.5 & 100 & 100 \\
\hline rtsn & 72.9 & 77.8 & 78.1 & 71.3 & 77.7 & 78.1 \\
\hline rtc & 63.5 & 69.2 & 69.2 & 55.7 & 70.2 & 68.1 \\
\hline rtcn & 56.0 & 59.3 & 59.3 & 55 & 62 & 61 \\
\hline rrbfs & 88 & 91.7 & 91.7 & 88.6 & 92.1 & 92.1 \\
\hline rrbfc & 92.1 & 97.3 & 97.3 & 94.5 & 97.9 & 97.9 \\
\hline wave 21 & 81.5 & 86.7 & 86.7 & 83.8 & 86.7 & 86.7 \\
\hline wave 40 & 82.1 & 86 & 86 & 80.6 & 86.4 & 86.4 \\
\hline led & 75.3 & 75.2 & 75.2 & 75 & 75.2 & 75.2 \\
\hline genF1 & 95.5 & 95.5 & 95.5 & 95.5 & 95.5 & 95.5 \\
\hline genF2 & 81.1 & 86.5 & 86.5 & 86.5 & 86.5 & 86.5 \\
\hline genF3 & 97.8 & 97.8 & 97.8 & 97.8 & 97.8 & 97.8 \\
\hline genF4 & 94.1 & 94.3 & 94.3 & 94.2 & 94.4 & 94.4 \\
\hline genF5 & 86.8 & 93.1 & 93.1 & 86.4 & 92.4 & 92.4 \\
\hline genF6 & 88.9 & 91.2 & 91.2 & 90.1 & 91.2 & 93 \\
\hline genF7 & 96.2 & 97.2 & 97.2 & 96.4 & 97.1 & 97.1 \\
\hline genF8 & 99.5 & 99.4 & 99.4 & 99.1 & 99.5 & 99.5 \\
\hline genF9 & 95.2 & 96.4 & 96.4 & 95.7 & 96.5 & 96.5 \\
\hline genF10 & 99.7 & 99.7 & 99.7 & 99.7 & 99.7 & 99.7 \\
\hline
\end{tabular}

The results given above of Hoeffding tree with two ways of predictions at leaves are compared with hoeffding option tree with option paths restricted to five.

But Hoeffding option tree has more accurate values than hoeffding trees in both cases with different prediction at leaves. HOT5 is more accurate in all environments than hoeffding trees.

The LED generator takes more time for evaluation when compared to other generators. The results in
$32 \mathrm{MB}$ and $400 \mathrm{MB}$ are quite similar but at an average, accuracy of hoeffding option trees is greater than the hoeffding trees

\section{CONCLUSION}

Data streams are defined and the evaluation of the Massive Online Analysis framework as a software environment for research on learning from evolving data streams and evaluating algorithms is done.

We used a prequential evaluation method to experimentally compare single classifier and ensemble data stream classifier which is considered as Hoeffding option trees. Use of option trees for classification in data streams is made and results are noted. Memory limits are set to define various environments where evaluation can be done.

Data generators which generate data sets based on some pattern are formed which are further used for evaluation and experiments are carried out on the given algorithms mainly on hoeffding trees and hoeffding option trees.

Majority class prediction and naïve Bayes prediction at the leaves is used for hoeffding trees and they are evaluated using the framework and the results are noted and accuracy of the algorithms mainly Hoeffding trees and Hoeffding option trees are compared for different data sets under various memory limits. It is observed that option nodes enable faster growth without instability in splitting decisions and have improved lookahead strategy.

As a future work, we plan to work on the evaluation method with other large number of algorithms to take a diversified look at the performance of the most recent stream mining techniques.

\section{REFERENCES}

[1]P. Domingos and G. Hulten, "Mining High Speed Data Streams", in Proceedings of the Association for Computing Machinery Sixth International Conference on Knowledge Discovery and Data Mining, 2000.

[2]P. Domingos and G. Hulten. A General Framework for Mining Massive Data Streams.

[3]Manish Mehta, Rakesh Agarwal, and Jorma Rissanen. "SLIQ : A fast scalable classifier for data mining". In Extending Database Technology, 1996.

[4]John Shafer, Rakesh Agarwal, and Manish Mehta. "SPRINT : A scalable parallel classifier for data mining “. In International Conference on Very Large Databases. 1996.

[5]Geoff Hulten, Laurie Spencer, and Pedro Domingos. Mining time-changing data streams. In $K D D$, pages 97-106, 2001.

[6]Eric Bauer and Ron Kohavi. An Empirical Comparison of Voting Classification Algorithms: Bagging, Boosting, and Variants

[7]Bernhard Pfarhringer, Goeffrey Holmes, and Richard Kirkby. "New Options for Hoeffding trees". 2007. 
[8]Ron Kohavi and Clayton Kunz, "Option Decision trees with majority votes". In International Conference on Machine Learning.

[9]Richard Kirkby, "Improving Hoeffding Trees", University of Waikato, 2007.

[10]Dariusz Brzezinski, "Mining data streams using concept drift”, Poznan University of Technolgy, 2010

Dr. P. Chenna Reddy did his B.Tech from S.V. University College of Engineering, Tirupati, M.Tech \& Ph.D from JNTU, Hyderabad. He has 15 years of Teaching experience. His areas of interest are Computer Networks and related fields. He is currently working on Bio inspired networking. He is currently working as Associate Professor at JNTUA College of Engineering, Pulivendula. He has published several papers in reputed journals and conferences.

B. Reshma Yusuf received her Bachelor degree with Computer Science and Engineering in 2010. She is been under Master of Technology degree with specialization in Computer Science and Engineering and a research scholar in JNTU, pulivendula. Her research interests include Data Mining and related fields. 